\title{
Philippe Raynaud: Le juge et le philosophe. Essais sur le nouvel âge du droit
}

\author{
Colin, 2009, 288 pp, ISBN: 978-2-200-35465-7
}

\author{
Olivier Camy
}

Published online: 27 April 2010

(C) Springer Science+Business Media B.V. 2010

Le livre de P. Raynaud réunit un ensemble d'articles déjà publiés dans le domaine de la philosophie du droit. Ces articles très variés couvrent un champ étendu. Les trois parties du livre en témoignent. Une première partie historique porte sur les doctrines du droit depuis l'Antiquité. Une deuxième partie est relative au rôle du juge, notamment dans le cadre américain. La dernière partie interroge les rapports entre droit et démocratie. Dans un premier temps, nous détaillerons le contenu de ces trois parties. Puis nous tenterons de dégager les lignes de force qui font l'intérêt essentiel de l'ouvrage.

La première partie intitulée "Aux origines de l'ordre juridique moderne" débute sur une réflexion relative à la Querelle des Anciens et Modernes; une querelle qui porterait essentiellement sur le rapport nature/loi et le rôle éducatif de la loi. Puis vient une confrontation utile entre la doctrine du droit de Kant et le droit révolutionnaire français. Il apparaît que Kant est plus proche des Lumières françaises (Turgot, Sieyès) que du modèle anglais. Kant tente notamment de redonner consistance au pouvoir exécutif tout en refusant la logique archaïsante du King in Parliament. Ce qui apparaît compliqué du fait qu'il promeut la primauté de la loi et d'un législatif réputé rationnel au détriment de la logique anglaise des cheks and balances; un dilemme qui était déjà celui des constituants français de 1791. P. Raynaud revient ensuite sur la doctrine française classique du droit public. Il fait apparaître la position paradoxale de cette doctrine sur la valeur juridique de la Déclaration de 1789. Les juristes qui accordent une valeur juridique à la Déclaration seraient aussi "les plus éloignés de l'esprit de la Révolution française" (p. 46). Ainsi Duguit et Hauriou affirment la supériorité de la Déclaration sur la Constitution de 1875 au nom d'une position jusnaturaliste implicite. Pour autant, ils refusent l'idée de droits subjectifs et universels. Quant aux juristes positivistes comme Carré de Malberg, ils s'accordent avec l'esprit de la Déclaration qui donne à la loi le soin de

O. Camy $(\square)$

IEP Paris et Université de Bourgogne, Paris, France

e-mail: olivier.camy@sciences-po.org 
garantir les droits de l'homme et du citoyen tout en refusant la valeur juridique des droits de 1789 puisqu'ils ne sont pas l'expression d'une loi positive ou étatique. Dans l'article suivant, P. Raynaud fait retour à la notion d'État de droit qui serait selon lui "problématique, sinon obscure" (p. 63). La filiation allemande (Rechtsstaat) conduit à la considérer comme un concept libéral et étatiste ayant fait l'objet de critiques tantôt pro ou anti démocratiques. D'un côté C. Schmitt rejette son côté "unilatéral" et dépolitisé. D’un autre côté, H. Kelsen dénonce sa faiblesse cognitive sur le fondement de l'identité État/droit, tout en approuvant son contenu libéral en tant que théoricien de la démocratie. La filiation anglaise (Rule of Law) nous renvoie à d'autres critères: la soumission de l'administration à la Common Law et les "qualités procédurales" du procès (p. 71). Enfin, P. Raynaud décline les variantes anglaise, américaine et française du constitutionnalisme moderne ainsi que leur destin commun; soit une démocratisation mais aussi le développement controversé de l'activisme judiciaire.

La deuxième partie intitulée "Figures du juge" débute par une réflexion sur la conception respective du juge chez Platon et Aristote. Platon insiste sur le rôle de "gardien des lois" mais aussi de magistrat du juge (en tant qu'il comble les lacunes de la loi). Mais il minore sa fonction proprement juridictionnelle dans le procès. Au contraire, Aristote valorise ce dernier rôle sans doute parce que, selon lui, le droit est “dit" à l'occasion d'un litige (p. 98). L'article suivant se concentre sur l'évolution de la figure du juge depuis l'Antiquité. Cette évolution démontre que la montée en puissance du juge est relativement tardive et fragile. Avec Aristote, le juge est étroitement soumis à la loi et à l'équité. Avec la modernité, le juge est contesté car il mettrait en danger "l'unité de la puissance du souverain" (p. 107). Et même la réhabilitation récente du pouvoir judiciaire reste ambivalente (en raison du conflit entre les théories rationaliste et irrationaliste de l'interprétation). L'article se termine par une mise en garde contre toute "conception angélique" du droit. Une mise en garde reprise dans l'article suivant qui traite du "juge et de la communauté" (p. 117). L'angélisme serait l'idée selon laquelle nous entrons dans une ère où le droit est principalement produit par le juge et donc s'émancipe de l'État, tandis que s'éclipse la figure du souverain. Mais la souveraineté est toujours vivante: elle continue de légitimer l'État, le droit et même le rôle du juge en tant que co-législateur. Elle révèle aussi "l'imperfection essentielle du droit international" (R. Aron). L'article suivant tente de démontrer que les doctrines actuelles relatives à la nature de la justice constitutionnelle sont en dette vis-à-vis de la pensée politique classique, notamment anglaise. Ainsi, la conception du juge de Locke est en partie retrouvée par Dworkin qui partage le même postulat rationaliste. Le juge, selon Locke, découvre la loi (par un acte de connaissance) sans se réclamer d'un droit naturel. De même, pour Dworkin, le juge ne ferait qu'appliquer la loi par une démarche immanente: il mettrait à jour des principes qui ne seraient ni transcendants, ni extérieurs. Quant à Hobbes, il refuse la fiction du juge comme "bouche de la loi" et fait de l'autorité (non de la vérité) la source de la loi. De ce point de vue, le scepticisme axiologique de M. Troper ainsi que sa théorie de l'interprétation comme acte de volonté sont bien des positions hobbesiennes. Les deux articles suivants enquêtent sur les controverses juridico-politiques relatives au judicaire américain. Le premier s'attache à montrer que les doctrines de "l'intention 
originaire" (Bork) et de la "constitution vivante" (Dworkin) s'opposent fondamentalement sur la nature du temps constitutionnel et de l'activité interprétative du juge. Pour autant elles ne renient pas les acquis de l'activisme judiciaire de la Cour suprême. La doctrine plus récente d'Ackerman ouvre une $3^{\text {ème }}$ voie qui tend à retrouver "les vertus oubliées de la souveraineté populaire" (p. 149). Le deuxième article explique le rôle que joue la référence à Marbury c. Madison dans la doctrine de l'intention originaire (Berger, Bork, Rehnquist). Cette doctrine conduit au moins à trois interprétations différentes de Marbury c. Madison qui ne permettent pas une description exacte du rôle du juge et ne font qu'idéaliser une des conceptions possibles des fondements de la Constitution américaine (soit la souveraineté du peuple et le gouvernement limité).

Enfin, la $3^{\text {ème }}$ partie intitulée "Le droit et la démocratie" s'ouvre par une réflexion consacrée à la nouvelle "démocratie juridique" (qui met en son centre le juge). P. Raynaud commence par formaliser les trois figures traditionnelles du juge: le magistrat qui tranche les litiges (Aristote), l'organe du souverain (Hobbes), le "découvreur" de règles applicables au cas particulier (Kant). Dans ces trois cas, le juge n'a pas d'autorité politique, contrairement à ce que postulent les théories contemporaines enclines à justifier la "dynamique des droits" subjectifs (dont la contrepartie serait un "déclin du droit"). Cette dynamique est à l'œuvre notamment chez Dworkin. Ce dernier tend en effet à promouvoir l' "utopie douce" d'une société donnant la priorité aux droits subjectifs et ayant exorcisé "l'élitisme de la représentation" ou l'autoritarisme de la démocratie de masse (p. 183). Une telle utopie présente, selon P. Raynaud, le risque du "conservatisme du progrès" et de la politisation du pouvoir judiciaire. La pratique américaine et française confirme l'existence de ces deux risques et conduit même à en concevoir un troisième: un contrôle accru des relations entre "individus moyens" au nom des droits des personnes ou des minorités (avec pour conséquence des frontières poreuses entre droit civil et droit pénal). L'article suivant revient sur ce triomphe des droits subjectifs qui s'est dessiné dès l'âge classique (Hobbes, Locke). Durant cet âge, les droits sont articulés avec un pouvoir souverain redéfini en raison du déclin de la fondation théologico-politique. Cela conduit à une "humanisation du droit" (p. 202). Succède à l'âge classique un âge moderne dont les caractéristiques reflètent une combinaison de la logique libérale du compromis avec la logique étatique de la volonté souveraine (au plan interne et international). Cette combinaison est aujourd'hui remise en cause par le modèle politique post-moderne dont les nouveaux principes de légitimité (notamment post-national) sont contestables. L'article suivant offre une réflexion sur "les modalités connues de fins de guerre civile et de sorties de la tyrannie" (p. 218). Parmi ces modalités, l'amnistie et la prescription, ont une origine grecque et romaine. Mais la prescription reste marquée par l' ancien droit car elle renvoie "au fondement contingent et indisponible" de l'autorité tandis que l'amnistie obéit à une logique plus moderne (volontariste) qui révèle "une imperfection constitutive du droit" (p. 222). Aujourd'hui, avec la disparition de la culture de guerre, amnistie et prescription ont le défaut de marginaliser le souci de justice et les droits des victimes. Une "dynamique indéfinie de la plainte" (p. 227) est apparue qui s'efforce de s'opposer à la logique de la souveraineté. Il reste que l'Afrique du Sud (Commission Vérité - Réconciliation) a 
inventé une voie nouvelle où 'l'amnistie n'autorise pas pour autant l'amnésie des dommages" (p. 228). Les deux articles suivants étudient la fondation sceptique de la démocratie; une solution originale revendiquée notamment par H. Kelsen. P. Raynaud étudie d'abord les affinités entre la théorie politique et le positivisme juridique de Kelsen. Mais il montre ensuite que le scepticisme de Kelsen a un rapport avec le droit naturel au sens où sa conception de la démocratie procède d'un "fondement naturel de l'idée de liberté" (p. 236). En cela, elle se rapproche du rousseauisme. Mais en articulant une philosophie pratique sceptique avec une conception égalitariste des droits, elle doit finalement plus à Hobbes. Il reste que le positivisme kelsenien est menacé par Kelsen lui-même lorsqu'il recommande l'instauration d'un contrôle de constitutionnalité. P. Raynaud reprend ici les objections de $\mathrm{M}$. Troper tendant à montrer que Kelsen adhère implicitement à une idéologie constitutionnaliste déjà formulée par Marshall (Marbury c. Madison). Cette idéologie est antipositiviste parce qu'elle est normative et surtout parce qu'elle ignore que "l'inconstitutionnalité est produite par le juge plutôt que constituée" (M. Troper). Si ce rôle du juge est vérifié, alors, toujours selon M. Troper, celui-ci doit être conçu plutôt comme un co-législateur situé au-dessus des représentants. Cela doit nous amener à repenser la théorie classique de la démocratie représentative. P. Raynaud croit cependant que cette théorie peut être conservée à condition de restaurer sa signification première qui est moins démocratique que libérale. La troisième partie se termine par un article intitulé "Vérité et pouvoir dans la politique moderne". P. Raynaud revient d'abord sur le modèle hobbesien qui articule une priorité absolue des droits subjectifs et un scepticisme moral. Ce modèle subvertit selon lui la doctrine rationaliste du Common Law (E. Coke). Il met en lumière une lignée hobbesienne allant de Hobbes à Austin jusqu'aux positivistes. H. Kelsen s'inscrit dans cette lignée tout en rejetant l'autoritarisme hobbesien. Il reste que la théorie démocratique relativiste des positivistes présente certains dangers analysés par Tocqueville. Dworkin et Habermas prolongent ces critiques en partant d'une structure argumentative qui est celle de la doctrine anglaise du Common Law.

Deux lignes de force apparaissent clairement dans ce recueil d'articles de P. Raynaud. La première est une tentative louable de redonner une place à la philosophie du droit tout en articulant celle-ci à la vieille philosophie politique et à la théorie du droit d'inspiration positiviste. Selon P. Raynaud, cela implique le rejet de deux conceptions de la philosophie du droit. La conception classique (ou métaphysique) qui tend à "fonder" le droit au risque du dogmatisme. Mais aussi la conception moderne "normative", déjà énoncée par Fichte, pour qui la philosophie elle-même ne peut survivre que comme une philosophie pratique productrice d'une communauté humaine régie par le droit. ${ }^{1} \mathrm{P}$. Raynaud adopte un troisième point de vue selon lequel la philosophie du droit aurait une tâche de "clarification" des notions utilisées principalement par la science du droit; cela dans une perspective historique permettant d'établir des filiations entre les controverses contemporaines et les contradictions de la philosophie du droit traditionnelle. Selon nous, une telle approche soulève au moins une difficulté. P. Raynaud tend à identifier la

\footnotetext{
${ }^{1}$ J. Rivelaygues, Leçons de métaphysique allemande, t. I, Grasset, 1990, p. 168.
} 
philosophie du droit à une épistémologie génétique qui réfléchit sur la façon dont l'objet juridique est constitué par la science du droit; cela sans oser se substituer à cette dernière. On réfléchit une fois que l'objet a été posé par l'activité de la science qui l'a constitué. Dès lors, la possibilité d'une véritable critique de l'ontologie positiviste dominante (seulement esquissée chez l'auteur) visant à identifier le droit à une technique d'exercice du pouvoir ou à encore à un édifice normatif est perdue. La possibilité d'un "philosopher" en droit reste sans doute encore à explorer, notamment grâce à la phénoménologie (dont on peut regretter qu'elle ne soit pas prise en compte dans l'ouvrage). ${ }^{2}$

La deuxième ligne de force du livre est la tentative de "modeliser" les positions théoriques des auteurs classiques et modernes de façon à faire apparaître des paradigmes juridico-politiques qui influencent telle ou telle argumentation contemporaine. Grâce à cette démarche, sont dégagées les antinomies d'une raison juridique historiquement située. Ce post-kantisme a l'avantage de nous donner une pédagogie extrêmement utile et éclairante de nos controverses contemporaines. Mais il a l'inconvénient de "positiver" ces controverses. En effet, la mise à jour d'antinomies permet de justifier tous les points de vue en présence, au moins de façon partielle, puisqu'ils ne sont pas considérés comme réellement contradictoires. ${ }^{3}$ Aucune des positions n'est fausse, erronée ou encore susceptible d'être dépassée. Ce post-kantisme a aussi l'inconvénient d'ignorer en partie la "déraison juridique" à l'œuvre dans nos sociétés contemporaines. Il ne prend pas en compte par exemple la question des camps où le juge est absent (mais où la norme règne entre le droit et le fait), de la bureaucratie (cautionnée par le droit de l'administration), du capitalo-communisme à la chinoise (organisé sans référence aux droits subjectifs et au juge constitutionnel), des rémanences théocratiques (parfois constitutionnalisées explicitement Cf. l'Iran); soit un ensemble de phénomènes qui participent pourtant à leur manière du "nouvel âge du droit".

\footnotetext{
${ }^{2}$ Elle se traduirait par une "pragmatie" et non une ontologie ou onto-théologie du droit (Husserl, le Heidegger de Sein und Zeit).

${ }^{3}$ On peut parler de dilemmes de la raison juridique en reprenant le titre de la thèse de P. Raynaud (Cf. P. Raynaud, M. Weber et les dilemmes de la raison moderne, Puf, 1987).
} 